\title{
Identification of Conditions for Nanostructured Burnishing and Subsurface Shear Instability
}

\author{
Viktor P. Kuznetsov ${ }^{1, \text { a) }}$, Sergey Yu. Tarasov ${ }^{2,3, \text { b) }}$, and Andrey I. Dmitriev 2, 4, c) \\ ${ }^{1}$ Kurgan State University, Kurgan, 640669, Russia \\ ${ }^{2}$ Institute of Strength Physics and Materials Science SB RAS, Tomsk, 634055, Russia \\ ${ }^{3}$ National Research Tomsk Polytechnic University, Tomsk, 634050, Russia \\ ${ }^{4}$ National Research Tomsk State University, Tomsk, 634050, Russia \\ a) Corresponding author: wpkuzn@mail.ru \\ b) tsy@ispms.tsc.ru \\ c) dmitr@ispms.tsc.ru
}

\begin{abstract}
Numerical as well as physical modeling of nanostructuring burnishing has been carried out to find out the process parameter limiting levels, which serve both to provide appropriate surface quality and positive deformationinduced structural modification of the subsurface layers as well as to avoid shear instability in the subsurface layers of burnished metal. The effects of load, burnishing speed, tool tip material, tool pass number and tribological transfer on the burnished surface roughness have been elucidated by the example of quenched and tempered steel 20X (EN 20Cr4). It was shown that overloading results in quasi-viscous flow of the subsurface material, deterioration of the surface and ruining the positive effect of nanostructuring burnishing.
\end{abstract}

Keywords: modeling, movable cellular automata, nanostructuring, burnishing, shear instability

\section{INTRODUCTION}

It has been shown that combination of normal and shear plastic strain induced in sliding provides severe plastic deformation [1] and fast generation of a nanosize subgrain layer in the subsurface of metals in sliding which may lead to shear instability of this layer with respect to shear stress [2]. The origin of such phenomenon is related to changing the deformation mechanism at the real contact areas from shear to grain boundary slipping in nanosize SPD-generated structures. This structurally modified and plasticized material is deformed as a quasi-viscous medium and has very strong adhesion to the counterpart thus causing galling and seizure. It is plain to see that the processed surface's quality may serve as an indicator to notice the shear instability onset which normally has to be avoided when presetting the burnishing process parameters. Another part of the story is that nanosize subgrains generated by sliding in the subsurface provide hardening due to Hall-Petch law and thus may reduce the wear rate of the component processed. So the choice is to generate the optimum thickness nanosize subgrain structure but avoid its instability against shear stress in the subsurface of metals.

The objective of this work is to establish the limiting nanostructuring burnishing process parameters by the criterion of shear instability onset in the subsurface material layer and workpiece-to-tool metal transfer. To achieve this, the numerical and physical modeling of the nanostructuring burnishing process was undertaken. The subsurface structural modification is determined by the power of the friction process, i.e. the energy delivered to the subsurface per time. Numerical modeling was devoted to studying the effect of loading on the subsurface structural modification while physical one to sliding speed and number of passes. 


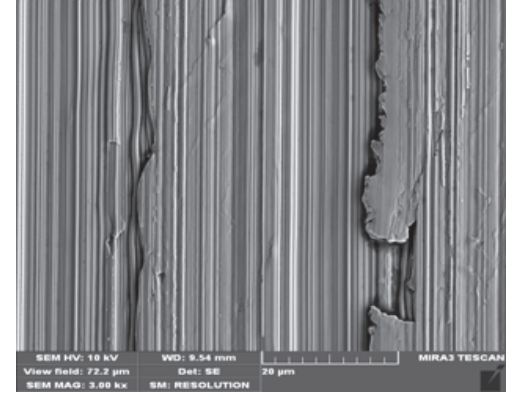

(a)

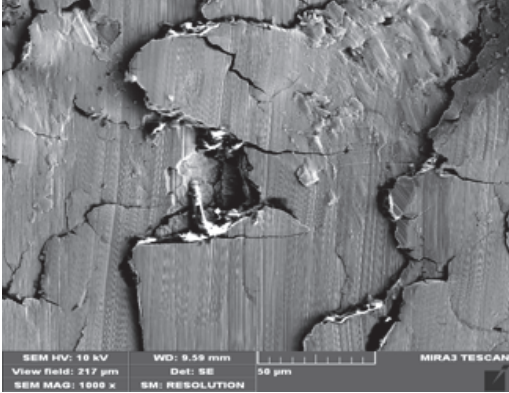

(b)

FIGURE 1. The SEM images of $20 \mathrm{X}$ steel surfaces burnished at $P=400$ (a) and $600 \mathrm{~N}$ (b)

\section{MATERIALS AND METHODS}

Steel samples have been fabricated of steel 20X (EN 20Cr4) in the shape of $80 \mathrm{~mm}$ diameter, $12 \mathrm{~mm}$ thickness disks. Steel samples have been carburized by gas to obtain $0.95 \mathrm{wt} \%$ concentration of carbon at the $0.1 \mathrm{~mm}$ depth below the surface. The final heat treatment was quenching $839^{\circ} \mathrm{C}$ in oil and tempering $250^{\circ} \mathrm{C}$ for $2 \mathrm{hr}$. The amount of retained austenite was 30 vol.\%. The hardness of the sample was HRC55.

Before nanostructuring burnishing, the samples had been precision turned by Sandvik WNGA 08048 hard metal cutters at cutting speed $v_{\mathrm{b}}=80 \mathrm{~m} / \mathrm{min}$ and feed rate $S_{\mathrm{T}}=0.06 \mathrm{~mm} / \mathrm{rev}$ for one tool setting block on Multus B300-W turning and milling center. The surface roughness was 0.34 and $0.76 \mu \mathrm{m}$, respectively.

The microstructure of the samples have been examined using TEM instrument JEOL JEM-2100, SEM instrument Tescan Mira 3 LMU with autoemission Schottky cathode and scanning autoemission electron microscope AURIGA CrossBeam. Surface roughness was determined using optical profilometer Wyko NT-1100.

\section{RESEARCH RESULTS}

Prior to nanostructuring burnishing under process conditions $F_{\mathrm{B}}=200 \mathrm{~N}, \mu=0.34, S_{\mathrm{B}}=0.04 \mathrm{~mm} / \mathrm{rev}, v_{\mathrm{B}}=$ $10 \mathrm{~m} / \mathrm{min}, n_{\mathrm{px}}=4$, steel 20X samples have been fine turned at $v_{\mathrm{T}}=80 \mathrm{~m} / \mathrm{min}, S_{\mathrm{T}}=0.06 \mathrm{~mm} / \mathrm{rev}$. The subsurface structure of the samples after nanostucturing burnishing has been modified so that the martensite needles were replaced for superfine deformation-induced structures.

The intensity of subsurface deformation in burnishing depends on the normal load value. This effect has been studied on 20X (HRC55) samples being burnished by DBN tool, at burnishing rate $10 \mathrm{~m} / \mathrm{min}$, feed rate $S_{\mathrm{B}}=$ $0.04 \mathrm{~mm} / \mathrm{rev}$ for a single tool pass. The SEM images in Fig. 1 show the burnished surfaces obtained at different normal load values. Almost smooth surface with some grooves for $P=400$ (Fig. 1(a)) and totally deteriorated surface for $P=600 \mathrm{~N}$ (Fig. 1(b)) can be seen. Too high normal load and, correspondingly, the friction force induces the shear instability which results in quasi-viscous flow and discontinuities of severely deformed subsurface layer thus giving the same burnished surface pattern as can be seen in Fig. 1(b), where such an effect has been achieved by strain accumulated at lower normal load but higher tool pass number.

\section{NUMERICAL MODELING OF THE NANOSTRUCTURING BURNISHING}

For numerical movable cellular automata modeling [3-6] a contact zone spot between two bodies in sliding with relative velocity transversely directed with respect to the compressive stress have been chosen.

The model included two blocks belonging to the bodied in sliding contact. The upper block was an indenter, while the lower one played a role of the steel to be burnished. The automatons of upper block have been loaded by external force which induced 1 to $10 \mathrm{MPa}$ stresses directed along the $O Y$ axis. On establishing the equilibrium, a transversal driving force has been applied in the direction of $O X$ to move the upper block at $V=0.5 \mathrm{~m} / \mathrm{s}$. The positions of the lower block automatons have been fixed to simulate the elastic response of the burnished material. 

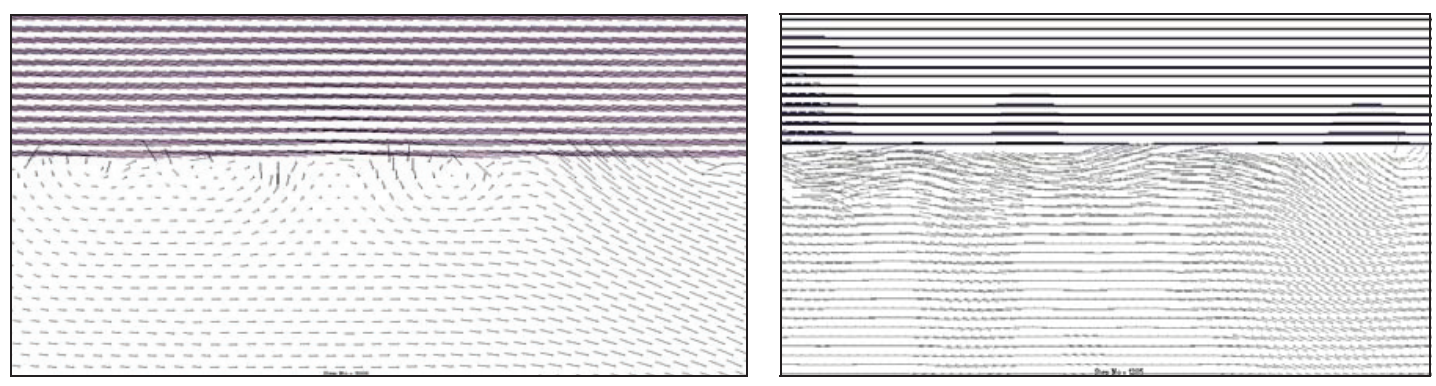

FIGURE 2. Fields of particle velocity near the specimen surface for two different moments of time

The loading parameters have been specified by doing so and then corresponded to severe and confined conditions of the sliding contact. In order to exclude the influence of the edge effects, periodical boundary conditions along the $O X$ axis were imposed, which simulated the dimension of the "assembly" generated. The length of the zone of interest was $0.1 \mathrm{~mm}$ for the automaton size as small as $1 \mu \mathrm{m}$. The number of automatons in the modeled fragment was above 9000. The physical characteristics of the indenter's and sample's automatons corresponded to those of superhard material and quenched to $\mathrm{HB} \geq 340-400$ steel, respectively. The initial roughness of the contacting surfaces has been simulated by pulling some automatons out of the superficial layer.

The computations demonstrate that on being brought in sliding contact, the asperities immediately start deforming and fracturing. Simultaneously, new linked pairs are created so that the result is the generation of a structurally modified subsurface layer under the loading. This structural modification penetrates only to $5-8 \mu \mathrm{m}$ depth below the surface unless corresponding normal stress is higher than $5 \mathrm{MPa}$. The steady-state configurations of the inter-automaton ties shows that linked pairs correspond to no-modification states and are marked by lines connecting the relevant automatons' center points. It can be seen that the zone where automata pair state switching occurs, the indenter/sample contact area stays localized for all time intervals.

Increasing the compressive stress up to $6 \mathrm{MPa}$ and even higher corresponds to applying the indenter of radius 2 $\mathrm{mm}$ and burnishing force $P_{\mathrm{B}} \geq 200 \mathrm{H}$ thus serving to intensify the subsurface structural modification. Structural evolution of the inter-automaton ties as well as strain intensity field for compressive stress level as high as $8 \mathrm{MPa}$ shows the thickness of the layer with broken inter-automaton ties is more than $10 \mu \mathrm{m}$. It is possible to distinguish two types of layers by typical structure of ties between the particles. The top $10 \mu \mathrm{m}$ thickness layer is in direct contacting with the indenter and here we can see its active structural modification. The interparticle ties are being broken and restored in this layer thus serving to develop processes of segmentation and intermixing. As before, the thickness of the layer is kept constant for the steady state motion.

The particle velocity field for a part of the sample below the free surface is shown in Fig. 2 for moments of time $t=1.108 \times 10^{6} \Delta t$ and $t=1.205 \times 10^{6} \Delta t$. In contrast to the smooth sliding regime Fig. 2 demonstrates us complex and non-linear behavior of the particles' motion in the vicinity of indenter/sample contract area. Such a mode of motion could be even called a "quasi-viscous" flow mode, which is a signature of shear instability in metals [6].

It should be noted that the inter-automaton tie breakdown criteria may be achieved even below the top subsurface layer but no intermixing action is observed in this case. The strain intensity field shows up here as a homogeneous one.

\section{DISCUSSION}

By modeling the effect of burnishing by a superhard indenter on subsurface deformation in sliding steel sample two different deformation modes which differ from each other by the strain intensity penetration depth and material flow pattern are found. The first mode is observed for compressive stress 1 to $5 \mathrm{MPa}$ and characterized by the strain penetration depth in the range 5 to $8 \mu \mathrm{m}$. The second mode shows total strain penetration to more than $10 \mu \mathrm{m}$ below the surface at compressive strength $8 \mathrm{MPa}$ with first $10 \mu \mathrm{m}$ of material being dragged and stirred by the indentor. The behavior of material particles in this layer is very similar to that of observed during sliding with seizure when a plasticized metal layer flows like a quasi-viscous nanocrystalline material under condition of shear instability [2]. In spite of the fact that such a treatment serves to produce nanosized subgrain structure and thus increase the hardness, 
the quasi-viscous flow would strongly affect the surface quality. Therefore, it would be a wise decision to avoid applying loading condition that lead to establishing such a mode.

In so doing, numerical simulation shows that overloading in burnishing results in structural changes of the intensity which depends on the strain degree and strain penetration below the surface. Two structurally different layers have been observed in numerical burnishing experiment as follows: (i) high strain layer of non-linear particle motion behavior or quasi-viscous flow and intermixing mode and (ii) underlying layer of less strain degree showing only the plastic deformation (broken ties) but no intermixing.

In physical modeling the behavior of subsurface metal under burnishing conditions deals with a large number of external and internal factors contributing to the process such as load, speed, friction coefficient, number of tool passes, temperature, adhesion, etc. Nevertheless, numerical modeling is capable of distinguishing between two modes of the material behavior during burnishing.

\section{CONCLUSION}

Overloading conditions in burnishing result in generation of a subsurface layer of non-linear quasi-viscous behavior. The origin of layer is similar with that of nanocrystalline layer obtained in tribological experiments under conditions of shear instability and intermixing and therefore could be called a tribological layer.

There is a consistency between the results of numerical and physical modeling in the sense of generation of shear instability motion in the subsurface of the steel in burnishing.

\section{ACKNOWLEDGEMENT}

This work has been carried out under financial support of the Program for Basic Scientific Research of the State Academy of Science on 2013-2020 and RFBR grants 13-08-00324, 13-08-98088 (S.Yu.T.) and 12-08-00960a, 1408-91330 (A.I.D.).

\section{REFERENCES}

1. S. Yu. Tarasov, D. V. Lychagin, and A. V. Chumaevski, Appl. Surf. Sci. C 274, 22 (2013).

2. S. Yu. Tarasov and V. E. Rubtsov, Phys. Solid State 53(2), 358 (2011).

3. W. Österle, A. I. Dmitriev, and H. Kloß, Faraday Discussions 156, 159 (2012).

4. S. G. Psakhie, E. V. Shilko, A. Yu. Smolin, A. V. Dimaki, A. I. Dmitriev, Ig. S. Konovalenko, S. V. Astafurov, and S. Zavshek, Phys. Mesomech. 14(5-6), 224 (2011).

5. A. I. Dmitriev and W. Österle, Tribology Lett. 53, 337 (2014).

6. A. I. Dmitriev, M. Schargott, and V. L. Popov, Wear 268(7-8), 877 (2010). 